appointed as vice-chancellors of many of Pakistan's universities, with adverse effects on the morale of academicians and researchers. None of the military governments made a serious effort to promote science. Against this background, it is surprising to read: "Worryingly, Pakistan's governance of its science seems all set to revert to the situation that prevailed under previous elected governments."

It would be interesting to compare the rise in the military budgets under military and elected governments with the budgets for education and health. Neither military nor elected governments have ever dared to reduce the military budget in real terms.

Muhammad Naim Siddiqi, Abdul Wahab Yusufzai Department of Psychiatry, Aga Khan University, PO Box 3500, Karachi 74800, Pakistan e-mail: naim.siddiqi@aku.edu

\section{Doping: world agency sets standards to promote fair play}

SIR - We at the World AntiDoping Agency (WADA) find parts of Donald Berry's Commentary 'The science of doping' (Nature 454, 692-693; 2008) potentially damaging to the fight against doping in sports.

WADA's accredited laboratories, including the French national laboratory LNDD (which handled the Floyd Landis case), must meet standards set by the International Standard for Laboratories (ISL) in validation methods, staff competency and chain of custody, for example. Compliance is assessed independently by bodies of the International Laboratory

Accreditation Cooperation.

Berry casts doubt on the laboratory component of the anti-doping procedure as a whole, but he fails to mention that the majority of the substances reported by the anti-doping laboratories are exogenous substances not naturally present in human beings. The development of testing procedures for endogenous substances includes samples from normal reference populations and from subjects administered with the substance under investigation, so that test-sample status and positivity criteria can be established. To determine cut-offs for the ratio of testosterone to epitestosterone $(T / E)$, tens of thousands of athlete samples were analysed to establish reference values. To detect exogenous administration of endogenous substances (such as pharmaceutical testosterone) by isotoperatio mass spectrometry (IRMS), validation is based on a combination of hundreds of known positive and negative samples analysed by many WADA anti-doping laboratories operating under the scrutiny of the ISL and of the International Organization for Standardization (such as ISO 17025).

Each sample test includes positive and negative qualitycontrol samples to assess the possibility of a false result. If these samples fail, the test must be repeated. An adverse analytical finding is not reported unless the quality criteria are met.

Laboratories participate in at least four rounds of blind and one double-blind proficiency test per year; the results of each round determine the accreditation status of the laboratory. False positives mean immediate revocation of accreditation.

Berry's implication that the results of $T / E$ ratio and IRMS analyses are interdependent is not altogether correct: according to the WADA list of prohibited substances, IRMS analysis stands alone as the basis of an exogenous testosterone finding. This is supported by legal precedents.

Contrary to Berry's suggestion that anti-doping tests may not be sufficiently specific, massspectrometry identification of exogenous substances relies on at least three diagnostic ions to avoid any interference or misidentification. For immunoassays, antibodies in the initial testing and confirmation procedures must have different antigen-epitope specificity. For analytes that are too small to have two independent antigenic epitopes, two different purification methods or two different analytical methods are used.

WADA encourages its accredited laboratories to publish in peer-reviewed journals. Although complete information cannot be released without compromising the efficacy of an anti-doping test, this is made available to legal panels on request.

WADA's mandate is not to foster "a sporting culture of suspicion, secrecy and fear", as suggested by your Editorial (Nature 454, 667; 2008), but to promote fair play by protecting clean athletes.

Arne Ljungqvist, Luis Horta, Gary Wadler World Anti-Doping Agency, Stock Exchange Tower, 800 Place Victoria, Suite 1700, PO Box 120, Montreal, Quebec H4Z 1B7, Canada e-mail:violet.maziar@wada-ama.org

\section{Doping: using flexible criteria could reduce false positives}

SIR - In his Commentary 'The science of doping' (Nature 454, 692-693; 2008), Donald Berry uses data from the Floyd Landis case as grounds for his claim that drug-testing practices are often based on flawed statistics. His concerns stand in contrast to the US Anti-Doping Agency's view of the outcome of the case (quoting from R. Mukhopadhyay and J. Griffiths Anal. Chem. 79, 8823-8825; 2007): "It's really easy to play Monday-morning quarterback and see an i that's not dotted or a that's not crossed, but that in no way undermined the validity or the reliability of the work that was done by the French lab."

This quote seems frivolous in the context of a class of drug-testing practices. These are based on separation of a sample's constituents by chromatography and detection of target compounds by mass spectrometry, one of the workhorses in anti-doping research. Identification is reported as positive when the test and reference sample signals agree within a particular tolerance window.

The size of this window is not constructed with an acceptable risk of false positives in mind. Rather, fixed decision criteria hold, regardless of the quality of the laboratory or the signal properties of the target compound. However, a laboratory that produces relatively precise results should deploy stricter criteria. Likewise, target compounds should be differentiated so that information in their signals can be respected.

Always deploying the same rigid criteria leads to a probability of false positives that depends on the particular laboratory and target compound in an undefined way. This situation is frustrating because the statistical solution - flexible criteria that account for various complications - was already published and thoroughly tested five years before these rigid criteria were introduced.

So, is it ignorance of the literature or failure to understand the analytical problem at hand that underlies the ongoing usage of these arbitrary decision rules? Laboratories, as well as their clients and (re-)accrediting organizations, should start reflecting on their accountability with regard to this avoidable malpractice.

N. M. Faber Chemometry Consultancy, Goudenregenstraat 6, 6573 XN Beek-Ubbergen, The Netherlands e-mail: nmf@chemometry.com

Contributions may be submitted

to correspondence@nature. com. Published contributions are edited. Issues of interest to authors are regularly featured at Nautilus (http://blogs.nature. com/nautilus), where we welcome comments and debate. 